\title{
High Energy Pulse Dynamics in Multimode GRIN Fibers
}

\author{
M. Zitelli ${ }^{1}$, F. Mangini ${ }^{2}$, M. Ferraro ${ }^{1}$, R. Crescenzi ${ }^{1}$, F. Frezza ${ }^{1}$, \\ D.S. Kharenko ${ }^{3}$, A. Niang ${ }^{2}$, S. Wabnitz ${ }^{1 ; 3}$ \\ 1Department of Information Engineering, Electronics and Telecommunications (DIET), \\ Sapienza University of Rome, Via Eudossiana 18, 00184 Rome, Italy. \\ 2Department of Information Engineering (DII), University of Brescia, Via Branze 38, 25123 Brescia, Italy. \\ 3Novosibirsk State University, Pirogova 1, Novosibirsk 630090, Russia. \\ mario.zitelli@uniroma1.it
}

\begin{abstract}
High energy, ultra-short multimode soliton pulse fission is observed and numerically studied in multimode GRIN fibers, showing complex dynamics bringing to multiple fundamental solitons that do not entirely follow standard single mode soliton perturbation theory predictions.
\end{abstract}

Keywords-Multimode fibers, Nonlinear optics

\section{INTRODUCTION}

Multimode (MM) optical fibers hold promise to increase the capacity of next-generation communications systems, and to scale up the energy of super-continuum and fiber laser sources. In this work, we consider the high-energy regime of femtosecond pulse propagation in a standard graded-index (GRIN) MM fiber. Multimode pulse fission at MW peak powers produces a train of fundamental solitons, which tend to conserve the same pulse width. Fundamental solitons must fulfil the condition

$$
N^{2}=\gamma_{j} P_{j} T_{0 j}^{2} /\left|\beta_{2 j}\right|=1,
$$

where, for the j-th soliton, $\gamma_{j}$ is the nonlinear coefficient, $P_{j}$ the peak power, $T_{0 j}$ the pulsewidth (over 1.763), and $\beta_{2 j}$ the dispersion, respectively. All parameters are evaluated at the specific wavelength of each soliton; $\gamma_{j}$ must account for an effective beam waist $w_{j}$, reduced with respect to the input as a consequence of periodic beam self-imaging. In order to reach the fundamental soliton condition, pulses with different peak powers red-shift their wavelength under the action of Raman nonlinearity and high order dispersion. As a consequence, the effective beam waist $w_{j}$ of the spatially self-imaging bullet corresponding to the $\mathrm{j}$-th soliton also increases, as the wavelength grows larger.

The Raman wavelength shift resulting from the fission process is much faster than the value predicted by the single mode soliton perturbation formula

This work was supported by the European Research Council (grant No. 740355), and by the Russian Ministry of Science and Education, (grant No. 14.Y26.31.0017).

$$
\Delta \lambda=\frac{4 \lambda_{j}^{2} \beta_{2 j} T_{R} Z}{15 \pi c T_{0 j}^{2}} .
$$

After the initial soliton wavelength multiplex is formed, solitons tend to conserve the initial common pulse width upon propagation, with different peak powers and effective waists. Wavelengths start shifting with slower speed during a second propagation phase, this time according to eq. 2 .

The multimode soliton fission dynamics in GRIN multimode fibers was experimentally studied, by launching high energy $150 \mathrm{fs}$ pulses at the $1550 \mathrm{~nm}$ input wavelength, for several input beam diameters. Pulse dynamics was also numerically investigated by using a vectorial $3 \mathrm{D}+1$ model, accounting for Kerr and Raman nonlinearity, dispersion up to 4-th order, polarization nonlinear coupling, wavelength dependent losses, nonlinear losses, and fiber index imperfections. Solitons with wavelengths up to $2300 \mathrm{~nm}$ have been observed, in some cases exhibiting a complex interaction dynamics, which limits their wavelength shift.

Nonlinear losses during the fission phase, and scattering of supercontinuum induced by periodic self-imaging are shown to be responsible for energy clamping at the fiber output.

\section{REFERENCES}

[1] W. H. Renninger et al., "Optical solitons in graded-index multimode fibres," Nat. Commun. 4, 1719 (2013).

[2] L. G. Wright, et al., "Spatiotemporal dynamics of multimode optical solitons," Opt. Express 23, 3492 (2015).

[3] 3. L. G. Wright, et al., "Controllable spatiotemporal nonlinear effects in multimode fibres," Nature Photonics 9, 306 (2015).

[4] 4. K. Krupa, et al., "Multimode nonlinear fiber optics, a spatiotemporal avenue," APL Photonics 11, 110901 (2019).

[5] 5. A. S. Ashan and G. P. Agrawal, "Spatio-temporal enhancement of Raman-induced frequency shift in graded-index multimode fibers," Opt. Lett. 44, 2637 (2019). 